\title{
Investigation of modified platelet-rich plasma (mPRP) in promoting the proliferation and differentiation of dental pulp stem cells from deciduous teeth
}

\author{
J. Wen ${ }^{1, *}$, H.T. Li ${ }^{2, *}$, S.H. $L i^{2}$, X. $L i^{3}$ and J.M. Duan ${ }^{2}$ \\ ${ }^{1}$ Guangdong Provincial Stomatological Hospital, Guangzhou, Guangdong Province, China \\ ${ }^{2}$ Department of Stomatology, Guangzhou General Hospital, Guangzhou Military Command, Guangzhou, \\ Guangdong Province, China \\ ${ }^{3}$ Department of Stomatology, Zhongshan City People's Hospital, Zhongshan, Guangdon Province, China
}

\begin{abstract}
Stem cells from human exfoliated deciduous teeth (SHEDs) have great potential to treat various dental-related diseases in regenerative medicine. They are usually maintained with $10 \%$ fetal bovine serum (FBS) in vitro. Modified platelet-rich plasma (mPRP) would be a safe alternative to $10 \%$ FBS during SHEDs culture. Therefore, our study aimed to compare the proliferation and differentiation of SHEDs cultured in MPRP and FBS medium to explore an optimal concentration of mPRP for SHEDs maintenance. Platelets were harvested by automatic blood cell analyzer and activated by repeated liquid nitrogen freezing and thawing. The platelet-related cytokines were examined and analyzed by ELISA. SHEDs were extracted and cultured with different concentrations of mPRP or $10 \%$ FBS medium. Alkaline phosphatase (ALP) activity was measured. Mineralization factors, RUNX2 and OCN, were measured by real-time PCR. SHEDs were characterized with mesenchymal stem cells (MSCs) markers including vimentin, CD44, and CD105. mPRP at different concentrations $(2,5,10$, and 20\%) enhanced the growth of SHEDs. Moreover, mPRP significantly stimulated ALP activity and promoted expression of RUNX2 and OCN compared with $10 \%$ FBS. mPRP could efficiently facilitate proliferation and differentiation of SHEDs, and $2 \%$ mPRP would be an optimal substitute for $10 \%$ FBS during SHEDs expansion and differentiation in clinical scale manufacturing.
\end{abstract}

Key words: mPRP; SHEDs; ALP; RUNX2; OCN

\section{Introduction}

Stem cells have shown high potential in dental-related regenerative medicine. Three critical factors, including seeding cells, scaffold materials, and osteogenesis-related differentiation factors, determine the effect and efficiency of tissue regeneration. Mesenchymal stem cells (MSCs) are considered ideal seeding cells for bone regeneration (1). A series of studies have demonstrated that bone marrow MSCs (BMMSCs) can differentiate into multiple cell types, such as skeletal tissue, adipocytes, and osteoblasts (2). However, BMMSCs extraction is still a complex process for oral tissue engineering. Thus, dental pulp stem cells (DPSCs) became a safer and easier substitute for BMMSCs. DPSCs were first isolated by Gronthos et al., and became a good candidate for tissue engineering (3). Other similar DPSCs include stem cells from human exfoliated deciduous teeth (SHEDs) first isolated by Miura et al., in 2003 (4). Compared with BMMSCs and DPSCs,
SHEDs have the highest proliferative capacity and multilineage differentiation potential $(4,5)$.

To culture the extracted SHEDs in vitro, fetal bovine serum (FBS) is the most commonly used culture supplement for MSCs, with multiple nutrition and growth factors, such as platelet-derived growth factors (6), insulin-like growth factors I and II (7), and TGF- $\beta$ (8). Nevertheless, compositions of FBS are too complex and may cause unexpected problems such as immunological rejection, infections by bovine virus and other pathogens, which limit its application in clinical trials (9). Platelet rich plasma (PRP), which is blood plasma enriched with platelets from autologous whole blood without exogenous antigens, could be a safe culture supplement and solve the above technical disadvantages (10). PRP contains a cocktail of growth factors, such as platelet-derived growth factor (PDGF), basic fibroblast growth factor (bFGF), insulinlike growth factor, transforming growth factor $\beta$ (TGF- $\beta$ ), and

Correspondence: J.M. Duan: <duanjianminmd@gmail.com>

${ }^{*}$ These authors contributed equally to this study.

Received March 17, 2016 | Accepted July 25, 2016 
vascular endothelial growth factor. All these growth factors play an important role in supporting and stimulating MSCs growth and expansion $(9,11,12)$. PRP has been widely applied in the field of oral regenerative treatment, such as maxillofacial bone defect repair and guided periodontal tissue regeneration (10). During treatment, SHEDs mixed with PRP are implanted into mandible defect areas to promote osseointegration and vascularization. A series of studies have confirmed that PRP can promote proliferation, migration and differentiation of mesenchymal stem cells, reduce the time of cell fusion, increase sizes of cell colonyforming units, maintain stem cells osteogenic, chondrogenic and adipogenic differentiation capacity, and maintain an immunosuppressive state $(10,13,14)$. Flow cytometry assays also showed that MSCs expressed high levels of PDGF-A, PDGF-B, bFGF, TGF- $\beta$ and IGF-1 receptors, suggesting their functional importance in MSCs maintenance $(15,16)$.

When DPSCs are cultured with PRP, its immunophenotype, colony formation unit (CFU) and directional differentiation ability remain unchanged. PRP promotes the proliferation and protein synthesis of DPSCs through PI3K/AKT, MAPK and $N F \kappa B$ signaling pathways activation (17). Furthermore, PRP induces DPSCs mineralization through upregulation of osteogenic genes and osteopontin (OPG) protein, enhancing alkaline phosphatase (ALP) activity (18).

Therefore, our hypothesis is that modified (m)PRP could be a safe alternative to FBS to promote SHEDs proliferation through multiple platelet-derived growth factors. In the present study, we successfully separated the $\mathrm{mPRP}$ and SHEDs with high purity. Thereafter, we evaluated the effects of different concentrations of mPRP on the proliferation and differentiation of SHEDs in comparison with $10 \%$ FBS. Furthermore, we studied multiple differentiation phenotypes and factors when SHEDs were supplied with differentiation medium containing different concentrations of mPRP and $10 \%$ FBS.

\section{Material and Methods}

All experimental procedures were carried out according to hospital regulations and medical ethics standards. Platelets: human platelets were collected from 4 male volunteers in the Blood Transfusion Department, Guangzhou General Hospital, Guangzhou Military Command. The volunteers were AB blood type, 18-35 years old, had good health status, and no family disease history. All volunteers gave written informed consent.

\section{Separation and activation of PRP}

Isolation of improved PRP: $10 \mathrm{~mL}$ platelets were extracted from volunteers and centrifuged at $1000 \mathrm{~g}$ for $20 \mathrm{~min}$ at room temperature to discard the supernatant. Heparin was added to platelets to a final concentration of $2 \mathrm{U} / \mathrm{mL}$. Four samples were mixed thoroughly and counted by a Hematology Analyzer (BC-3000, Shenzhen Mindray Bio-Medical Electronics Co., LTD, China). The platelet concentration was adjusted to approximately $10^{12} / \mathrm{L}$ for PBS and for mPRP. Improved PRP activation: PRP was aliquoted into vials, immersed in liquid nitrogen for 5 min and quickly warmed at $37^{\circ} \mathrm{C}$ for $5 \mathrm{~min}$, three times (10). Vials were then centrifuged at $1000 \mathrm{~g}$ for $20 \mathrm{~min}$ at room temperature and platelet sediment was collected through a $0.2 \mu \mathrm{m}$ filter. The samples were stored at $-80^{\circ} \mathrm{C}$ until use.

\section{Measurement of PDGF-AA and TGF- $\beta 1$ by ELISA}

The levels of PDGF-AA and TGF- $\beta 1$ were measured by ELISA kit (R\&D, USA). Serial dilution of PDGF-AA and TGF- $\beta 1$ were prepared on a 96-well plate, for standardization. The concentrations of the growth factors were determined based on a standard curve. Each test was done with triplicate wells.

\section{Isolation and culture of SHEDs}

Teeth. After parents signed the informed consent form, two mandibular caries-free lower central deciduous incisors from 6 - to 10-year-old healthy children were extracted by a dentist. The pulp tissue was removed and cultured to obtain dental pulp stem cells according to the literature (4). Briefly, the teeth were placed in precooled $\alpha$-MEM (Gibco, USA) immediately after removal and sterilized in $75 \%$ ethanol. To extract the primary stem cells, high-speed dental handpieces were used along the cementoenamel junction to grind a groove without breaking through to the pulp, within $4 \mathrm{~h}$. After $75 \%$ ethanol disinfection and repeated PBS washing, the deciduous incisors were split along the groove. Pulp tissue was extracted with a barbed broach, cut into pieces and digested with $1: 13 \mathrm{~g} / \mathrm{L}$ collagenase and $4 \mathrm{~g} / \mathrm{L}$ neutral protease at $37^{\circ} \mathrm{C}$ for $1 \mathrm{~h}$. Dental pulp stem cells pellets were suspended with $\alpha$-MEM, $20 \%$ FBS. Cells $\left(2 \times 10^{5}\right)$ were seeded onto a 6 -well plate and cultured in $37^{\circ} \mathrm{C}$ in a $5 \% \mathrm{CO}_{2}$ incubator (Heraeus, Germany) for 3 days. $\alpha$-MEM with $20 \%$ FBS was applied to the culture of separated SHEDs for two passages then changed to $10 \%$ FBS. When the cells reached $90 \%$ confluence, they were transferred to a T25 flask. SHEDs were passed for 3 to 4 times and each passage was determined when reaching $70 \%$ confluence.

\section{Cell proliferation assay}

Third passage SHEDs $\left(2 \times 10^{3}\right)$ were seeded on 96well plates and cultured for 1 to 7 days. A cell growth curve was drawn based on a CCK-8 cell counting kit (Dojindo, Japan) by measuring $450 \mathrm{~nm}$ absorbance. To determine the effect of different concentrations of PRP on SHEDs proliferation, the fourth passage of SHEDs was seeded on 96-well plate supplemented with $\alpha$-MEM containing 2, 5 , 10 , and $20 \%$ PRP or $10 \%$ FBS. The cells were then cultured for 7 days. Everyday, $10 \mu \mathrm{L} \mathrm{CCK}-8$ was added and absorbance at $450 \mathrm{~nm}$ was measured.

\section{Characterization of SHEDs}

SHEDs morphology observation. Third passage SHEDs were digested and diluted to the concentration 
of $1 \times 10^{7} / \mathrm{L}$. Cells were seeded on 6 -well plates with polylysine pre-treated coverslips until they adhered onto the slips. Slips were removed and the cells were stained with hematoxylin and eosin to observe cell morphology under a microscope.

Immunocytochemical detection and phenotypic characterization. Third passage SHEDs on coverslips were fixed with $4 \%$ formalin for $2 \mathrm{~h}$. To eliminate endogenous peroxidase activity, the coverslip was incubated with $10 \%$ hydrogen peroxide for $10 \mathrm{~min}$. After blocking with $3 \%$ normal goat serum for $30 \mathrm{~min}$, the fixed cells were stained with mouse anti-human cytokeratin (or vimentin) antibody, followed by treatment with the biotinylated secondary antibody. Then, the cells were stained with hematoxylin, dehydrated with xylene, and sealed with neutral gum. The cell phenotype was detected with DAB staining and observed under a microscope.

Flow cytometry assay. Fourth passage SHEDs were fixed and stained with CD34, CD44, and CD105 antibodies to check SHEDs surface markers.

\section{SHEDs in vitro differentiation}

Third passage SHEDs $\left(1 \times 10^{5}\right)$ were seeded onto 6 -well plates. When cells reached a $70 \%$ confluence, cell mineralized nodules and lipid droplets formation capacity were tested after adding mineralization-inducing medium (final concentration of $50 \mu \mathrm{g} / \mathrm{L}$ ascorbic acid, $10 \mathrm{mmol} / \mathrm{L}$ $\beta$-glycerophosphate and $0.01 \mathrm{mmol} / \mathrm{L}$ dexamethasone) or adipogenic induction medium (final concentration of $1 \mu \mathrm{mol} / \mathrm{L}$ dexamethasone, $10 \mu \mathrm{mol} / \mathrm{L}$ insulin, $200 \mu \mathrm{mol} / \mathrm{L}$ indomethacin, $0.5 \mathrm{mmol} / \mathrm{L}$-isobutyl-methylxanthine). After culturing for 30 days, cells were fixed with formalin and stained with Alizarin red or oil red-O to separately test the mineralization and adipogenesis capacities of SHEDs.

\section{ALP enzyme activity test}

Fourth passage SHEDs were seeded $\left(5 \times 10^{3}\right)$ onto 96-well plates. Every 2, 4, and 6 days, ALP kit (Jiancheng, China) was applied and $520 \mathrm{~nm}$ absorbance was measured for the detection of ALP activity using the Enzyme Activity Reader (Biocell, USA).

\section{RNA extraction and real-time PCR}

Total RNA was extracted by Trizol. cDNA was prepared through reverse transcription. Primers were synthesized by Shanghai Shenggong, China. PCR primer sequences are as follows: hRUNX2, forward: 5'- TCCA CACCATTAGGGACCATC-3', reverse: 5'-TGCTAATGCT TCGTGTTTCCA-3'; OCN, forward: 5'-GGCAGCGAGG TAGTGAAGAGA-3', reverse: 5'-CTCCTGAAAGCCGAT GTGG-3'; hGAPDH, forward: 5'-GACAACTTTGGCATCG TGGA-3', reverse: 5'-ATGCAGGGATGATGTTCTGG-3'. GAPDH was set as the internal control. After the realtime PCR, RUNX2 and OCN mRNA levels were calculated and compared among the different SHEDs groups.

\section{Data analysis}

Experiments were repeated three times and statistical analysis was carried out using GraphPad (GraphPad Software Inc., USA) or SPSS software (SPSS Inc., USA). One-way ANOVA was used to test the difference among different groups in each time-point. To test the interaction between time and groups, two-way factorial ANOVA was applied. Multiple comparisons were based on the LSD method. The results were considered to be significant when $\mathrm{P} \leqslant 0.05$.

\section{Results}

\section{Phenotypic characterization showed that SHEDs are similar to MSC}

HE staining of SHEDs showed that the major morphology of SHEDs was typically spindle-like, while a few cells had polygonal or oval nucleus. The result suggested that most of SHEDs were stem cells mixed with other cell types (Figure 1A). To further validate whether these SHEDs were stem cell-like, two cell markers, cytokeratin and vimentin, were examined by immunochemistry. The cell marker of differentiated epithelial cells, cytokeratin was negative (Figure $1 \mathrm{~A}$ ). On the other hand, the immune staining of vimentin was strongly expressed in the nucleus of SHEDs (Figure 1A). During third passage culture, SHEDs entered the exponential growth phase on the second day and stationary phase on the 6th to 7th day based on the growth curve (Figure 1B), indicating that extracted SHEDs had a high proliferation potential. Cell surface markers CD34, CD44 and CD105 were checked by flow cytometry. Ninety-nine percent of the cells were CD44positive; $90.13 \%$ were CD105-positive; all of the cells lacked hematopoietic markers CD34 (Figure 1C), further confirming the high proliferation potential. The above results demonstrated that the SHEDs were mostly MSCs.

\section{Extracted SHEDs had high differentiation potential}

Subsequently, we assessed SHEDs in vitro osteogenic and adipogenic differentiation. SHEDs were harvested and treated with mineralization-inducing medium. During the first six days, the cells grew rapidly and gradually overlapped each other. On the 30th day, clear distinct nodules were detected in the center of the cell and Alizarin red staining was positive under a microscope (Figure 2A). Consistent results were obtained in the cell adipogenesis assay; the SHEDs formed a clear bright point with positive oil red-O staining on the 21st day after adipogenesis-inducing medium culture (Figure 2B). The results demonstrated that SHEDs were not only proliferative but also had a high differentiation potential.

\section{Effect of different concentrations of MPRP on SHEDs proliferation}

Improved rich PRP was a pale yellow liquid. Platelet concentration was $10^{12} / \mathrm{L}$, about five times higher than normal. mPRP was enriched with various growth factors. 


\section{A H\&E}

Cytokeratin

Vimentin

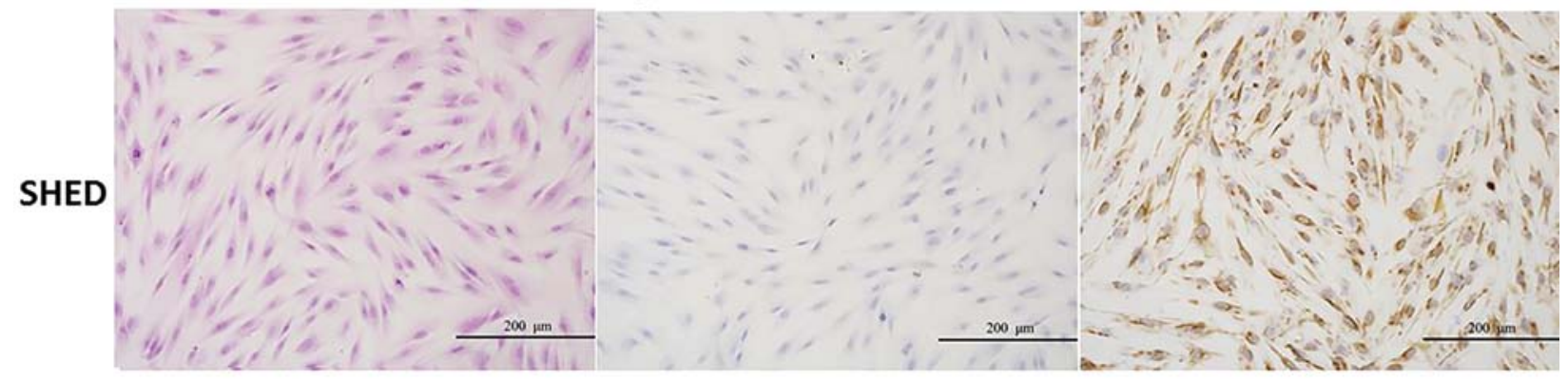

B
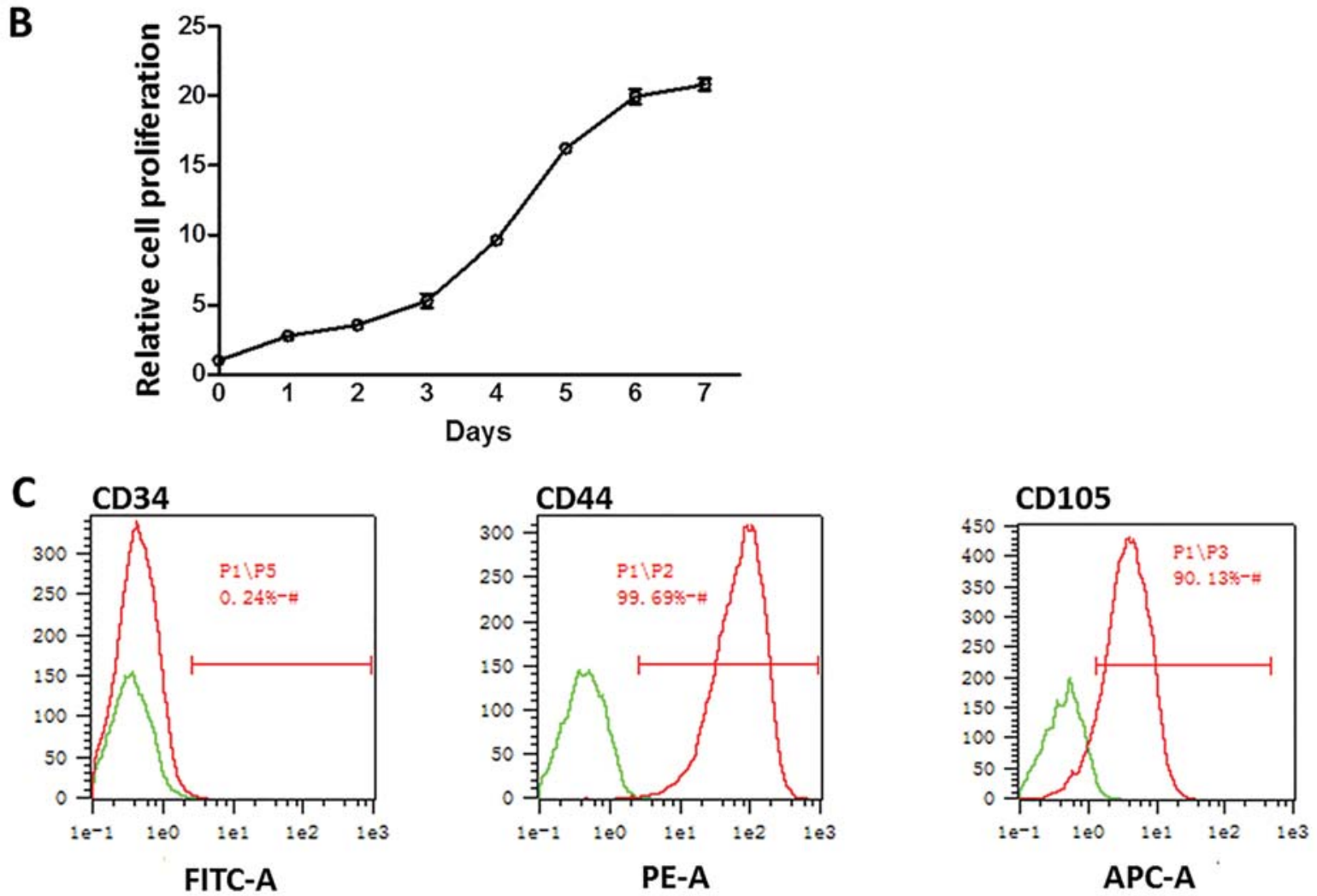

Figure 1. Photomicrographs of extracted stem cells from human exfoliated deciduous teeth (SHEDs) showing characteristics of mesenchymal stromal cells (spindle-like shape) with high proliferation activity. A, H\&E staining of SHEDs and immunocytochemical staining of cell markers cytokeratin, vimentin, $\times 200$. B. SHEDs cell proliferation was quantified by CCK-8 assay for 1-7 days. Data are reported as mean percentages \pm SD from three independent experiments. $C$, Flow cytometry analysis of cell surface markers CD34, CD44, and CD105.

The concentration of PDGF-AA was $19.159 \mu \mathrm{g} / \mathrm{L}$ and the concentration of TGF- $\beta 1$ was $57.163 \mu \mathrm{g} / \mathrm{L}$ (data not shown). Figure $3 \mathrm{~A}$ shows the effect of different concentrations of mPRP on SHEDs proliferation. At the beginning of cell culture, $2,5,10$, and $20 \%$ of mPRP had no significant improvement on SHEDs proliferation compared with the control group. From day 3 to day 5 , SHEDs in all groups entered the exponential growth phase. Two percent
mPRP showed a similar promotion effect as 10\% FBS. The proliferation for $5 \% \mathrm{mPRP}$ was a little lower than that of $2 \% \mathrm{mPRP}$ and $10 \%$ FBS. However, 10 and $20 \% \mathrm{mPRP}$ exhibited a much weaker proliferation $(P<0.05)$ when compared with the control, 2 and 5\% mPRP. When the cells reached a stationary phase on day 6, 2 and $5 \%$ mPRP-treated groups had a similar number of SHEDs similar to that of the $10 \%$ FBS group, but 10 and $20 \%$ 

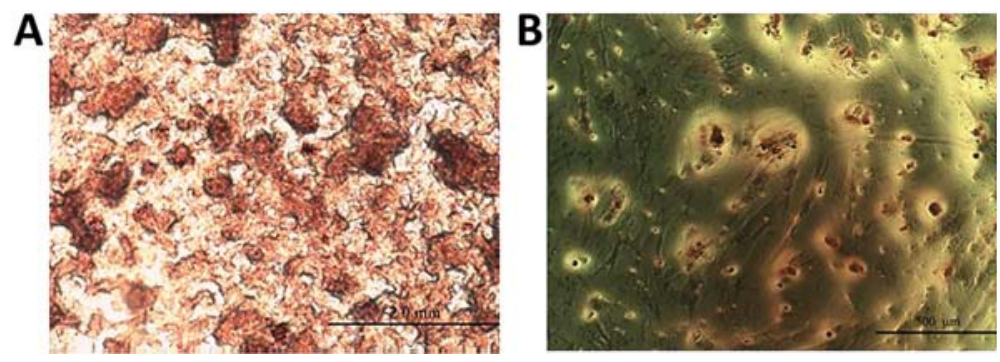

Figure 2. Stem cells from human exfoliated deciduous teeth presented a high differentiation capacity during adipogenesis and mineralization. $A$, cells after 30 days treatment with mineralization induction medium. Cells were stained with Alizarin red, $\times 10$. $B$, cells after 21 days treatment with adipogenesis induction medium. Cells were stained with oil red-O, $\times 100$.
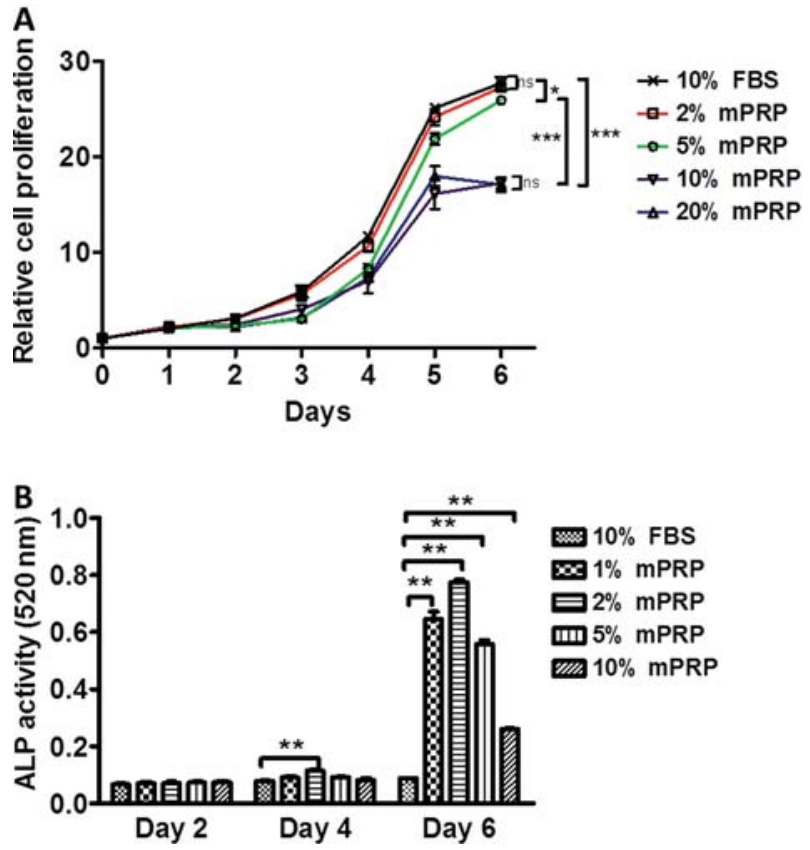

Figure 3. Effect of different concentrations of modified plateletrich plasma (mPRP) on cell proliferation $(A)$ and ALP activity of stem cells from human exfoliated deciduous teeth $(B)$. Data are reported as means $\pm S D$ from three independent experiments. ${ }^{*} \mathrm{P}<0.05$, ${ }^{* *} \mathrm{P}<0.01,{ }^{* * *} \mathrm{P}<0.001$ vs control group (10\% FBS) (Student's t-test)

mPRR had less cells compared with the above groups. These results indicated that different concentrations of mPRP improved SHEDs proliferation dose-dependently. Two percent mPRP seems to be the ideal culture concentration for SHEDs growth.

\section{Effect of different concentrations of mPRP on SHEDs mineralization}

ALP is an important component during SHEDs mineralization. Thus, we determined the levels of SHEDs mineralization by checking ALP activity. Although different concentrations of MPRP did not show a superior promoting effect on SHEDs proliferation than $10 \%$ FBS, they caused a striking upregulation of ALP activity on the 6th day of SHEDs culture $(P<0.01$; Figure $3 B)$.
A concentration increase from 1 to $2 \% \mathrm{mPRP}$ activated the ALP activity. This increase reached a peak at $2 \% \mathrm{mPRP}$, as a concentration increase from 2 to $10 \%$ decreased the ALP activity. Compared with day 2 and day 4, day 6 SHEDs showed the highest ALP activity, which suggests that when SHEDs reach confluence at stationary phase, the cells enter the mineralization differentiation period.

\section{Effect of different concentrations of mPRP's on SHEDs differentiation factors}

RUNX2 and OCN are two key factors that lead to MSC osteoblast differentiation. Therefore, we focused on these genes during SHEDs osteoblastic differentiation induced by mPRP treatment. Real-time PCR results showed that on day 7 after mineralization media induction, 1, 2, and $5 \%$ mPRP significantly upregulated the mRNA expression of RUNX2 compared with the control group. A concentration of $10 \% \mathrm{mPRP}$ conversely repressed the RUNX2 levels (Figure 4A). A similar result was obtained for OCN mRNA levels, which were significantly induced in the 1,2 and $5 \%$ mPRP groups, but not in the 10\% mPRP group compared with the control group (Figure 4B).

\section{Discussion}

Even though MPRP has been widely used in clinical applications for decades because of its enriched autologous growth factors and secretory proteins, few studies have focused on its influence on SHEDs proliferation and differentiation. Furthermore, little has been done to find a better alternative to $10 \%$ FBS when culturing SHEDs.

In the present study, we successfully isolated and purified mPRP with high quality. As several reports have shown, PRP effects vary among individuals due to agerelated systemic feedback mechanisms and different serum supplements $(19,20)$. Therefore, we mixed four batches of mPRPs to reduce individual variation. Platelets from $A B$ blood type were used to minimize antibody components, which could repress agglutination caused by immune rejection. To reduce the contamination of other cell components during mPRP separation, multifunctional cell separator was applied to the collected mPRP. 

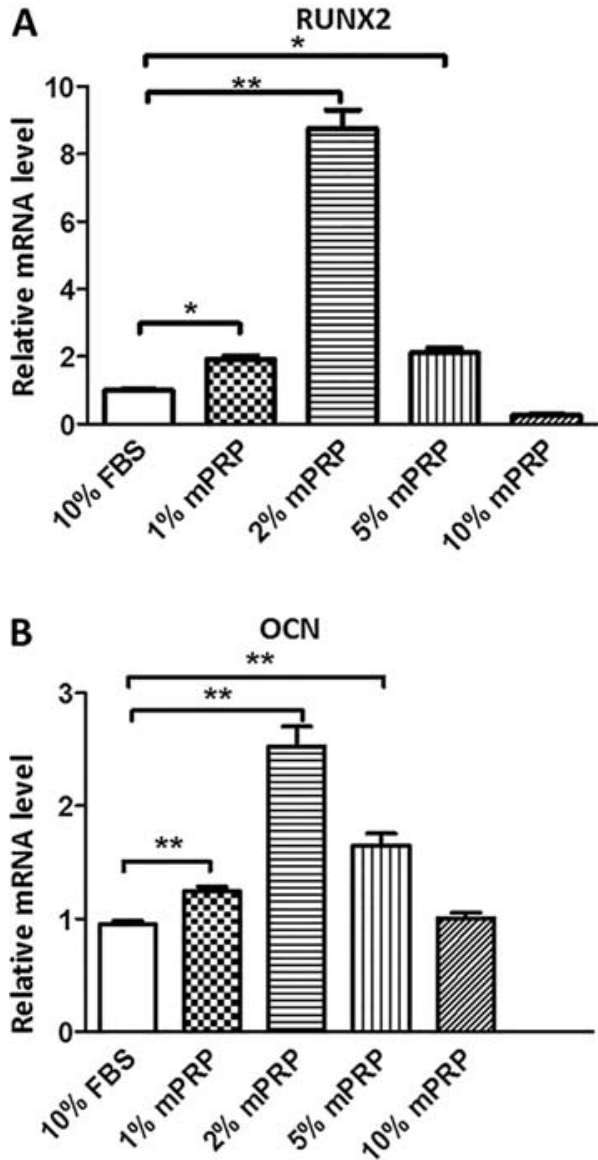

Figure 4. Effect of different concentrations of modified plateletrich plasma (mPRP) on mRNA expression of RUNX2 $(A)$ and OCN $(B)$ in stem cells from human exfoliated deciduous teeth (SHEDs) cells, by quantitative real-time PCR. ${ }^{*} \mathrm{P}<0.05$, ${ }^{* *} \mathrm{P}<0.01$ vs control group (Student's t-test).

Moreover, we also utilized the improved mPRP activation technology by repeated liquid nitrogen freezing and thawing. The various growth factors present in activated $\mathrm{mPRP}$ play important roles in cell proliferation, chemotaxis, and angiogenesis.

The extracted SHEDs were characterized with stem cell-like morphology. The cell growth curve proved that SHEDs were highly proliferative. Immunohistochemical results showed that SHEDs were MSC-like, cytokeratinnegative and vimentin-positive. Consistently, flow cytometry assay also showed that SHEDs were positively stained with MSC cell surface markers, CD44 and CD105. In vitro differentiation assay showed that SHEDs have a high potential to differentiate into osteoblasts and adipocytes.

To evaluate the possibility of replacing FBS by MPRP as a culture supplement during SHEDs growth and differentiation, we demonstrated that PRP promotion effect on SHEDs proliferation and differentiation was dosedependent. The experiments proved that $2 \% \mathrm{mPRP}$ had the optimal effect on the stem cells' proliferation. We also showed that an excessively high concentration of MPRP would impair the promotion effect. In a former study, we have explained that such a phenomenon is a result of prostaglandin E2 release by MPRP. Low concentrations of prostaglandin E2 can promote SHEDs proliferation while high levels will inhibit it (13). Another alternative explanation is related to the antiplatelet growth factor component in the plasma $(10,21)$. Flow cytometry assay showed that MSCs expressed high levels of these growth factor receptors, such as platelet-derived growth factor $A$ and $B$ and TGF- $\beta 1$ receptors (9). The above growth factors can activate multiple SHEDs signaling pathways, such as $\mathrm{PI} 3 \mathrm{~K}$ and $\mathrm{NF} \kappa \mathrm{B}$, further promoting cell regeneration and inhibiting apoptosis $(1,17,22,23)$. A detailed screening on PRP components demonstrates that platelet-derived growth factor and insulin-like growth factor-1 promote cell proliferation; acidic fibroblast growth factor, insulin-like growth factor 1 and insulin-like growth factor 2 promote extracellular matrix synthesis; TGF- $\beta$, platelet-derived growth factor, acidic fibroblast growth factor and basic fibroblast growth factor are involved in DPSCs odontoblast differentiation $(9,14,24)$.

Upregulation of OPG and ALP occurs during MSCs osteogenic differentiation (18). If mPRP differentiation medium could also promote osteogenic differentiation of SHEDs, its use in tissue engineering has full potential, presenting the desired effect and avoiding risks such as infection and immune rejection. ALP activity is an important indicator for osteoblast cell differentiation and maturation, reflecting the level of mineralization ability and osteogenic transformation (25). Our result suggests that different concentrations of MPRP can enhance ALP activity of SHEDs, with an optimized concentration of $2 \%$. RUNX2, which belongs to RUNX transcriptional factor family, is a major gene that regulates a large number of critical genes during osteoblastic differentiation and skeletal morphogenesis. OCN, one of the targets of RUNX family, is an osteoblast-specific protein which is essential for bone cell maturation. Shen et al. (26) has found that in vitro culture of SHEDs can express osteoblast markers, such as RUNX2, OCN, and bone sialoprotein. As in former reports, we have confirmed the upregulation of RUNX2 and OCN during SHEDs osteogenesis. These results indicate that during SHEDs differentiation, mPRP had an advantage over $10 \%$ FBS through enhancement of ALP activity and upregulation of mineralization factors, RUNX2 and OCN.

We showed that MPRP contains a high concentration of PDGF-AA and TGF- $\beta 1$. mPRP dose-dependently improved SHEDs proliferation similar to $10 \%$ FBS and it has a superior function in promoting SHEDs osteogenesis. Notably, as SHEDs would not cause antigen-induced immune rejection, they could be applied in allograft and in 
dental tissue engineering. MPRP could serve as an alternative to replace FBS in in vitro culture and differentiation of SHEDs, which could hopefully resolve the current challenge in SHEDs amplification and improve the clinical safety during dental regenerative therapy.

\section{References}

1. Huang YC, Leung VY, Lu WW, Luk KD. The effects of microenvironment in mesenchymal stem cell-based regeneration of intervertebral disc. Spine J 2013; 13: 352-362, doi: 10.1016/j.spinee.2012.12.005.

2. Malgieri A, Kantzari E, Patrizi MP, Gambardella S. Bone marrow and umbilical cord blood human mesenchymal stem cells: state of the art. Int J Clin Exp Med 2010; 3: 248-269.

3. Gronthos S, Mankani M, Brahim J, Robey PG, Shi S. Postnatal human dental pulp stem cells (DPSCs) in vitro and in vivo. Proc Natl Acad Sci U S A 2000; 97: 13625-13630, doi: 10.1073/pnas.240309797.

4. Miura M, Gronthos S, Zhao M, Lu B, Fisher LW, Robey PG, et al. SHED: stem cells from human exfoliated deciduous teeth. Proc Natl Acad Sci U S A 2003; 100: 5807-5812, doi: 10.1073/pnas.0937635100.

5. Huang GT, Gronthos S, Shi S. Mesenchymal stem cells derived from dental tissues vs those from other sources: their biology and role in regenerative medicine. $J$ Dent Res 2009; 88: 792-806, doi: 10.1177/0022034509340867.

6. Childs CB, Proper JA, Tucker RF, Moses HL. Serum contains a platelet-derived transforming growth factor. Proc Natl Acad Sci U S A 1982; 79: 5312-5316, doi: 10.1073/ pnas.79.17.5312.

7. Honegger A, Humbel RE. Insulin-like growth factors I and II in fetal and adult bovine serum. Purification, primary structures, and immunological cross-reactivities. J Biol Chem 1986; 261: 569-575.

8. Roberts AB, Anzano MA, Wakefield LM, Roche NS, Stern DF, Sporn MB. Type beta transforming growth factor: a bifunctional regulator of cellular growth. Proc Natl Acad Sci U S A 1985; 82: 119-123, doi: 10.1073/pnas.82.1.119.

9. Anitua E, Prado R, Orive G. Safety and efficient ex vivo expansion of stem cells using platelet-rich plasma technology. Ther Deliv 2013; 4: 1163-1177, doi: 10.4155/tde. 13.68.

10. Bielecki T, Dohan Ehrenfest DM. Platelet-rich plasma (PRP) and Platelet-Rich Fibrin (PRF): surgical adjuvants, preparations for in situ regenerative medicine and tools for tissue engineering. Curr Pharm Biotechnol 2012; 13: 1121-1130, doi: $10.2174 / 138920112800624292$.

11. Assoian RK, Grotendorst GR, Miller DM, Sporn MB. Cellular transformation by coordinated action of three peptide growth factors from human platelets. Nature 1984; 309: 804-806, doi: $10.1038 / 309804 a 0$.

12. Stoika RS. [Phylogenetic characteristics of the structure and function of polypeptide growth factors localized in the blood platelets of mammals]. Zh Evol Biokhim Fiziol 1989; 25: 373-379.

13. Duan J, Kuang W, Tan J, Li H, Zhang Y, Hirotaka K, et al. Differential effects of platelet rich plasma and washed platelets on the proliferation of mouse MSC cells.

\section{Acknowledgements}

This research was supported by the Science and Technology Planning Project of Guangdong Province, China (\#2011B031800201).

Mol Biol Rep 2011; 38: 2485-2490, doi: 10.1007/s11033010-0385-7.

14. Li H, Usas A, Poddar M, Chen CW, Thompson S, Ahani B, et al. Platelet-rich plasma promotes the proliferation of human muscle derived progenitor cells and maintains their stemness. PLoS One 2013; 8: e64923, doi: 10.1371/journal. pone.0064923.

15. Massague J. Transforming growth factors. Isolation, characterization, and interaction with cellular receptors. Prog Med Virol 1985; 32: 142-158.

16. Kraus MH, Pierce JH, Fleming TP, Robbins KC, Di Fiore PP, Aaronson SA. Mechanisms by which genes encoding growth factors and growth factor receptors contribute to malignant transformation. Ann N Y Acad Sci 1988; 551: 320-335, doi: 10.1111/j.1749-6632.1988.tb22358.x.

17. Peng $Y$, Huang $S$, Wu $Y$, Cheng $B$, Nie $X$, Liu $H$, et al. Platelet rich plasma clot releasate preconditioning induced PI3K/AKT/NFkappaB signaling enhances survival and regenerative function of rat bone marrow mesenchymal stem cells in hostile microenvironments. Stem Cells Dev 2013; 22: 3236-3251, doi: 10.1089/scd. 2013.0064

18. Huang FM, Yang SF, Zhao JH, Chang YC. Platelet-rich fibrin increases proliferation and differentiation of human dental pulp cells. J Endod 2010; 36: 1628-1632, doi: 10.1016/ j.joen.2010.07.004.

19. Lohmann M, Walenda G, Hemeda H, Joussen S, Drescher $\mathrm{W}$, Jockenhoevel $\mathrm{S}$, et al. Donor age of human platelet lysate affects proliferation and differentiation of mesenchymal stem cells. PLoS One 2012; 7: e37839, doi: 10.1371/ journal.pone.0037839.

20. Niemeyer P, Fechner K, Milz S, Richter W, Suedkamp NP, Mehlhorn AT, et al. Comparison of mesenchymal stem cells from bone marrow and adipose tissue for bone regeneration in a critical size defect of the sheep tibia and the influence of platelet-rich plasma. Biomaterials 2010; 31: 3572-3579, doi: 10.1016/j.biomaterials.2010.01.085.

21. Dohan Ehrenfest DM, Bielecki T, Mishra A, Borzini $P$, Inchingolo $F$, Sammartino G, et al. In search of a consensus terminology in the field of platelet concentrates for surgical use: platelet-rich plasma (PRP), platelet-rich fibrin (PRF), fibrin gel polymerization and leukocytes. Curr Pharm Biotechnol 2012; 13: 1131-1137, doi: 10.2174/13892011 2800624328

22. Suzuki H, Iso Y. Clinical application of vascular regenerative therapy for peripheral artery disease. Biomed Res Int 2013; 2013: 179730 , doi: $10.1155 / 2013 / 179730$.

23. Das $\mathrm{R}$, Jahr $\mathrm{H}$, van Osch GJ, Farrell $\mathrm{E}$. The role of hypoxia in bone marrow-derived mesenchymal stem cells: considerations for regenerative medicine approaches. Tissue Eng Part B Rev 2010; 16: 159-168, doi: 10.1089/ten.teb. 2009.0296 . 
24. Nakashima M. The effects of growth factors on DNA synthesis, proteoglycan synthesis and alkaline phosphatase activity in bovine dental pulp cells. Arch Oral Biol 1992; 37: 231-236, doi: 10.1016/0003-9969(92)90093-N.

25. Daltaban O, Saygun I, Bal B, Balos K, Serdar M. Gingival crevicular fluid alkaline phosphatase levels in postmenopausal women: effects of phase I periodontal treatment. $J$ Periodontol 2006; 77: 67-72, doi: 10.1902/jop.2006.77.1.67.

26. Shen Y, Yang S, Liu J, Xu H, Shi Z, Lin Z, et al. Engineering scaffolds integrated with calcium sulfate and oyster shell for enhanced bone tissue regeneration. ACS applied materials \& interfaces 2014; 6: 12177-12188, doi: 10.1021/am501448t. 